\title{
Thermodiffusion of interacting colloids. I. A statistical thermodynamics approach
}

\author{
Jan K. G. Dhont ${ }^{\mathrm{a})}$ \\ Forschungszentrum Jülich, IFF/Weiche Materie, D-52425 Jülich, Germany
}

(Received 22 July 2003; accepted 21 October 2003)

\begin{abstract}
The thermal diffusion coefficient of colloids consists of two additive contributions, one related to specific interactions between the surfaces of colloidal particles with solvent molecules, and a contribution due to interactions between the colloidal particles. In the present paper, the effect of intercolloidal particle interactions on their thermodiffusive behavior is discussed within a statistical thermodynamics framework. Transport coefficients are expressed in terms of the interaction potential between the colloidal spheres. A special feature of macromolecular systems is that this interaction potential is a potential of mean force, which is temperature dependent. It is shown that under certain conditions this implicit temperature dependence gives rise to negative Soret coefficients, that is, to diffusion of macromolecules to hot regions. () 2004 American Institute of Physics. [DOI: 10.1063/1.1633546]
\end{abstract}

\section{INTRODUCTION}

Diffusive mass transport induced by temperature gradients, the so-called "Soret effect,", 1,2 has been described within the framework of irreversible thermodynamics. ${ }^{3-5}$ Mass transport within such an approach is formulated in terms of Onsager coefficients. So far, for dense systems limited work has been done to express such Onsager coefficients for temperature-gradient induced diffusive mass transport in terms of molecular interaction potentials. For molecular mixtures, where interaction potentials are inherently temperature independent, Bearman et al. ${ }^{6}$ derived microscopic expressions for the Soret coefficient on the basis of the binary Liouville equation. A similar microscopic approach, based on the Smoluchowski equation for a single polymer chain, has been used by Khazanovich ${ }^{7}$ to describe thermodiffusion of a single polymer chain. As shown in the subsequent paper, however, his Smoluchowski equation does not include certain explicit temperature-gradient contributions, but assumes the applicability of the Smoluchowski equation for a homogeneous system to systems with temperature variations. In any case, Khazanovich's theory describes diffusion of single polymer chains, not interacting with other polymers. The same temperature-gradient contributions to the Smoluchowski equation are not accounted for in Streater's type of approach, ${ }^{8}$ nor is the temperature dependence of the potential of mean force recognized.

More recently, Luettmer-Strathmann ${ }^{9}$ analyzed thermodiffusion of noninteracting polymers by means of a regular solution grid model, partly by simulations. Uphill thermodiffusion, that is, diffusion to hot regions, is found for certain combinations of the polymer-polymer, solvent-solvent, and polymer-solvent interaction energies. The molecular mechanism is probably similar to the sign change of the thermal diffusion coefficient in binary fluids on changing composition. ${ }^{10-14}$ Single colloidal particle thermodiffusion

${ }^{\text {a)} E l e c t r o n i c ~ m a i l: ~ j . k . g . d h o n t @ f z-j u e l i c h . d e ~}$ has also been considered theoretically for magnetic/charged colloidal spheres by Morozov. ${ }^{15,16}$ Again, uphill diffusion is predicted under certain conditions.

Uphill thermodiffusion of macromolecules was recently found experimentally for polymer solutions and colloidal dispersions of Boehmite rods ${ }^{17}$ and protein solutions, ${ }^{18}$ while for magnetic particles the thermal diffusion coefficient strongly decreases on applying an external magnetic field, depending on the orientation of the magnetic field relative to the temperature gradient. ${ }^{19}$ These experiments are done on very dilute systems, where interactions between macromolecules are not important. The only published data on macromolecular systems I am aware of, where interaction effects are discussed, are on a micellar system ${ }^{20}$ and solutions of polystyrene in ethyl acetate ${ }^{21}$ and in toluene. ${ }^{22}$ Strong interaction effects on the thermal diffusion coefficient have been observed here, where the concentration dependence of the Soret coefficient for the micellar system is reversed on adding surfactant. This shows that interactions can have a pronounced effect on thermodiffusive behavior.

No microscopic expressions have been derived for the concentration dependence of the thermal diffusion coefficient of colloids due to intercolloidal interactions. Furthermore, the temperature dependence of the colloidal pair-interaction potential, being a potential of mean force, in some cases probably plays an important role in the diffusive behavior of these systems. It is the aim of the present paper to derive a microscopic expression for the thermal diffusion coefficient for interacting, spherical macromolecules, where the temperature dependence of the pair-interaction potential is accounted for. The temperature dependence of the pairinteraction potential is shown to give rise to unusual, negative Soret coefficients under certain conditions.

Diffusion of spherical colloidal particles in a given, time-independent temperature gradient will be considered. The thermal diffusion coefficient as calculated for such a 
time-independent temperature profile is identical to the diffusion coefficient that one would employ to describe processes where the temperature is allowed to vary with time. A time-independent temperature profile is imposed in a thermal diffusion forced Rayleigh scattering (TDFRS) experiment. Here, a standing interference pattern is imposed on the sample by crossing two laser beams. A trace amount of absorbing dye is added to the solvent, which then gives rise to a stationary, sinusoidal temperature profile (for an overview of experimental techniques used to detect the Soret effect, see Ref. 23). The diffusion of colloidal particles is very slow in comparison to heat conduction by the solvent, so that a change in the concentration of colloidal particles does not affect the temperature profile. The temperature $T(\mathbf{r})$ at position $\mathbf{r}$ is written as

$$
T(\mathbf{r})=T+\Delta T(\mathbf{r}),
$$

where $T$ is the mean temperature of the system under investigation, and the superimposed sinusoidal profile is given by

$$
\Delta T(\mathbf{r})=A_{T} \sin \left\{k_{0} z\right\},
$$

where $A_{T}$ is the amplitude of the temperature variation. The $z$ direction is defined as the direction in which the temperature variation extends. The wavelength $\lambda_{T}$ of the temperature grating is set by the wave vector $k_{0}$ as $\lambda_{T}=2 \pi / k_{0}$.

The aim here is to derive, from thermodynamic and statistical mechanical considerations, an equation of motion for the number density $\rho(\mathbf{r}, t)$ of colloidal particles of the "irreversible thermodynamics form"

$$
\frac{\partial}{\partial t} \rho(\mathbf{r}, t)=D \nabla^{2} \rho(\mathbf{r}, t)+D_{T} \nabla^{2} T(\mathbf{r}),
$$

where $D$ is the collective mass diffusion coefficient and $D_{T}$ is the thermal diffusion coefficient. The definition of the thermal diffusion coefficient used here differs from definitions sometimes used by others, in the sense that sometimes instead of $D_{T}$ one uses $\rho D_{T}$ in Eq. (3), while other combinations are also used. Within the present approach, both $D$ and $D_{T}$ will be expressed in terms of integrals involving the interaction potential between the colloidal particles.

Single particle thermodiffusion due to specific, temperature-dependent interactions of the surface of the colloidal spheres with solvent molecules is briefly considered in Sec. VI. This contribution to $D_{T}$ should be added to the expressions for $D_{T}$ as derived in the present paper.

The equation of motion (3) is easily solved for an initial state (at time $t=0$, say) where the number density is a constant, independent of position. For small temperature variations, that is, for small enough amplitudes $A_{T}$ in Eq. (2), the response of the density will be linear, so that

$$
\rho(z, t)=\rho+\Delta \rho(z, t),
$$

with $\rho$ the mean colloidal number density, and

$$
\Delta \rho(z, t)=F(t) \sin \left\{k_{0} z\right\},
$$

where $F(t=0)=0$. Substitution into Eq. (3) then leads to a differential equation for $F(t)$ which is easily solved, leading to

$$
\Delta \rho(z, t) / \rho=S_{T} \Delta T(z)\left[\exp \left\{-D k_{0}^{2} t\right\}-1\right],
$$

where the Soret coefficient $S_{T}$ is defined as

$$
S_{T}=\frac{D_{T}}{\rho D} .
$$

The dimension of $S_{T}$ is $1 / K$. In literature, various definitions of the Soret coefficient can be found, according to the various possible definitions for $D_{T}$, as discussed above. In the stationary state, according to Eq. (6) we have

$$
\Delta \rho(z, t \rightarrow \infty) / \rho=-S_{T} \Delta T(z),
$$

implying that there is net colloidal mass transport to cold regions when $S_{T}>0$, and to hot regions when $S_{T}<0$. Both of these cases have been observed experimentally for various macromolecular systems, as discussed before.

In binary fluid mixtures of components $A$ and $B$, say, "the Soret coefficient of $A$ " is usually defined to be positive when $A$ diffuses to cold regions. This implies that "the Soret coefficient of $B$ " is negative, since $B$ migrates to hot regions. In the present case of binary mixtures of solvent and colloidal particles, the driving force for diffusion is due to the colloidal particles. We shall therefore refer to "the Soret coefficient" as the coefficient for the colloids.

This paper is organized as follows: In Sec. II, thermodynamic arguments in combination with force balance on the Brownian time scale are shown to lead to expressions for the collective and thermal diffusion coefficient in terms of the osmotic pressure. The connection between the osmotic pressure and the potential of mean force is made in Sec. III. Since the temperature dependence of the potential of mean force plays such an important role for the thermodiffusive behavior of colloids, the notion of mean force will be discussed in some detail. The collective and thermal diffusion coefficient are expressed in terms of the pair-interaction potential between the colloids, up to first order in concentration, in Sec. IV. A model calculation for short-ranged potentials to leading order in concentration is presented in Sec. V. It is shown that the temperature dependence of the potential of mean force can give rise to diffusion to hot regions, that is, to "uphill thermodiffusion," when the potential is strongly attractive and short ranged, and the depth of the attractive potential increases with increasing temperature. In Sec. VI, some remarks are made on thermodiffusion induced through specific interactions between the surface groups of colloidal spheres and solvent molecules.

\section{THERMODYNAMIC DERIVATION OF THE MASS TRANSPORT EQUATION}

When the temperature profile (1) and (2) is applied at time $t=0$ say, the originally homogeneous suspension with number density $\rho$ will become inhomogeneous. Assuming small deviations of the temperature $\Delta T(\mathbf{r})$ and small gradients, we shall derive an equation of motion of the type (3) for the spatially varying contribution $\Delta \rho(\mathbf{r}, t)$ of the colloidal particle number density. Here, we allow for spatial variations in all three dimensions.

The equation of motion for the number density $\rho(\mathbf{r}, t)$ of colloidal spheres is a conservation equation 


\begin{tabular}{|ll|ll|}
\hline $\mathrm{T}$ & Box 1 & $\mathrm{T}+\delta \mathrm{T}$ & Box 2 \\
$\mathrm{V}$ & & $\mathrm{V}$ & \\
$\mathrm{Nc}, 1$ & & $\mathrm{Nc}, 2$ & \\
$\mathrm{Ns}, 1$ & & $\mathrm{Ns}, 2$ & \\
\hline
\end{tabular}

(a)

\begin{tabular}{|c|c|c|}
\hline $\begin{array}{l}T \\
V \\
N c, 1-\delta N c \\
N s, 1-\delta N_{s}\end{array}$ & & $\begin{array}{l}\mathrm{T}+\delta \mathrm{T} \\
\mathrm{V} \\
\mathrm{Nc}, 2+\delta \mathrm{Nc} \\
\mathrm{Ns}, 2+\delta \mathrm{Ns}\end{array}$ \\
\hline
\end{tabular}

$$
\frac{\partial}{\partial t} \rho(\mathbf{r}, t)=-\nabla \cdot[\mathbf{v}(\mathbf{r}, t) \rho(\mathbf{r}, t)],
$$

where $\nabla$ is the gradient operator with respect to position $\mathbf{r}$, and $\mathbf{v}$ is the translational velocity of a sphere. On the Brownian time scale, where inertial forces are very small, there is a balance of all noninertial forces acting on a spherical colloidal particle. ${ }^{24,25}$ There are two essentially different kinds of such forces: the hydrodynamic force $\mathbf{F}^{h}$ that a colloidal sphere experiences due to friction with the solvent once it attains a nonzero translational velocity, and the remaining force $\mathbf{F}$ due to direct interactions with other colloidal spheres. Force balance implies that

$$
\mathbf{0}=\mathbf{F}(\mathbf{r}, t)+\mathbf{F}^{h}(\mathbf{r}, t) .
$$

With the neglect of hydrodynamic interactions between the colloidal particles, the hydrodynamic force is equal to

$$
\mathbf{F}^{h}(\mathbf{r}, t)=-\gamma \mathbf{v}(\mathbf{r}, t),
$$

where $\gamma=6 \pi \eta_{0} a$ is the friction coefficient of the sphere with radius $a$ in a solvent with shear viscosity $\eta_{0}$. Hence, once an expression for $\mathbf{F}$ is found in terms of gradients in density and temperature, the equation of motion (3) can be found from Eqs. (9)-(11), leading to explicit expressions for $D$ and $D_{T}$.

In order to obtain an expression for the interaction force $\mathbf{F}$ in a system subjected to a temperature gradient, it is essential to be able to assume local equilibrium. The dispersion is divided into small volume elements, here after referred to as "boxes." Each box can be treated as a homogeneous, thermodynamic system in equilibrium if

intensive variables vary on a length scale that is very much larger than the linear dimension of each box; and

(ii) the linear dimension of the boxes is much larger than the range $R_{D}$ of direct interactions between the colloidal particles.

The first condition implies that mass transport is very slow in comparison to relaxation times of fluctuations within a box and that within each box intensive variables are constant, independent of position. Hence, $\lambda_{T}=2 \pi / k_{0}$ should be very much larger than $R_{D}$. The second condition then implies that each box may be regarded as a thermodynamic system in internal equilibrium.
Note that in a colloidal system, heat transport occurs primarily through the solvent, certainly at low volume fractions of colloids. It is therefore feasible that relatively large gradients in temperature still allow one to assume local equilibrium (of the subset of colloidal particles), as compared to molecular fluids.

It should be noted that in a TDFRS experiment, heat is continuously added to the system (by absorption of light by the dye molecules, as explained in the Introduction). A stationary temperature profile is established because heat is conducted out of the dispersion to the surroundings, primarily by the solvent, at the same rate as heat is added. As for mass transport, the rate of heat conduction is slow for long wavelengths as compared to equilibration times within the boxes, so that local equilibrium remains assured.

Consider two neighboring boxes " 1 " and " 2 ," with prescribed equal volumes $V$ and with temperature $T$ and $T$ $+\delta T$, respectively. The number of colloidal spheres in box 1 and 2 is $N_{c, 1}$ and $N_{c, 2}$, and the number of solvent molecules $N_{s, 1}$ and $N_{s, 2}$, respectively. We shall ask for the reversible work $\delta w^{\text {rev }}$ necessary to move $\delta N_{c}$ colloidal spheres and $\delta N_{s}$ solvent molecules from box 1 to box 2 , at prescribed temperatures of the two boxes [see Fig. 1(a)]. This process is assumed reversible, complying with the local equilibrium assumption during mass transport as discussed above. The reversible work $\delta w^{\text {rev }}$ necessary to achieve the abovedescribed mass transport from box 1 to box 2 is equal to $\delta F+S_{1} \delta T_{1}+S_{2} \delta T_{2}$, where $\delta F$ is the accompanying change in Helmholtz free energy of the sum of the two boxes, $S_{j}$ is the entropy, and $\delta T_{j}$ the change of temperature of box $j .{ }^{26}$ Since the temperature of both boxes remains constant during mass transport, the latter, entropic contributions are absent. The work is thus equal to the change $\delta F$ of the free energy. The Helmholtz free energy of each of the two boxes is a function of the number of solvent molecules and colloidal particles in the box, its volume, and the temperature. The reversible work necessary for the above described process is equal to [see Fig. 1(a)]

$$
\begin{aligned}
\delta w^{\mathrm{rev}}= & \delta F \\
= & F\left(N_{c, 1}-\delta N_{c}, N_{s, 1}-\delta N_{s}, V, T\right) \\
& +F\left(N_{c, 2}+\delta N_{c}, N_{s, 2}+\delta N_{s}, V, T+\delta T\right) \\
& -F\left(N_{c, 1}, N_{s, 1}, V, T\right)-F\left(N_{c, 2}, N_{s, 2}, V, T+\delta T\right) .
\end{aligned}
$$


Since the dispersion is space filling, we have in each of the two boxes (with $j=1$ or 2 )

$$
v_{s}^{0} N_{s, j}+v_{c}^{0} N_{c, j}=V,
$$

where $v_{s}^{0}$ and $v_{c}^{0}$ are the volumes occupied by a solvent molecule and a colloidal sphere, respectively. Due to the very large size of a colloidal sphere as compared to that of the solvent molecules, virtually all solvent molecules are surrounded by other solvent molecules as in the pure solvent. For incompressible solvents, the specific molar volume of solvent will therefore be assumed independent of colloid concentration and pressure. The temperature dependence of the molar volume of solvent is not essential to the physics of thermodiffusion, and will therefore also be neglected. In order to indicate the independence of the molar volume $v_{s}$ of solvent on intensive variables, it is denoted with a superscript "0." The specific molecular volume of a colloidal sphere is to very good approximation equal to its bare volume, and will be denoted as $v_{c}^{0}$. It is also assumed to be independent of concentration, pressure, and temperature. ${ }^{27}$ A prescribed volume $V$ thus implies that $\delta N_{s}=-\left(v_{c}^{0} / v_{s}^{0}\right) \delta N_{c}$. Expanding the free energies with respect to $\delta N_{s}$ and $\delta N_{c}$, using that $\partial F\left(N_{c}, N_{s}, V, T\right) / \partial N_{c}$ $=\mu_{c}\left(N_{c}, N_{s}, V, T\right)$, with $\mu_{c}$ the chemical potential of the colloidal particles, and similarly for the solvent, it is thus found that

$$
\begin{aligned}
\delta w^{\mathrm{rev}} / \delta N_{c}= & \mu_{c}\left(N_{c, 2}, N_{s, 2}, V, T+\delta T\right) \\
& -\mu_{c}\left(N_{c, 1}, N_{s, 1}, V, T\right) \\
& -\frac{v_{c}^{0}}{v_{s}^{0}}\left\{\mu_{s}\left(N_{c, 2}, N_{s, 2}, V, T+\delta T\right)\right. \\
& \left.-\mu_{s}\left(N_{c, 1}, N_{s, 1}, V, T\right)\right\} .
\end{aligned}
$$

The continuum form of Eq. (14) reads

$$
\delta w^{\mathrm{rev}} / \delta N_{c}=\mathbf{L} \cdot \nabla\left[\mu_{c}(\mathbf{r}, t)-\frac{v_{c}^{0}}{v_{s}^{0}} \mu_{s}(\mathbf{r}, t)\right],
$$

with $\mathbf{L}$ the distance over which a colloidal sphere is moved on average on transport from box 1 to 2 , that is, $\mathbf{L}$ is equal to $\mathbf{R}_{2}-\mathbf{R}_{1}$, with $\mathbf{R}_{j}$ the center position of box $j$. Note that both the temperature and concentration variations are accounted for in the above spatial gradients. Since $\delta w^{\text {rev }} / \delta N_{c}$ $=-\mathbf{F} \cdot \mathbf{L}$, with $\mathbf{F}$ the force with which surrounding material acts on a colloidal sphere (which has to be counterbalanced quasistatically by the force needed to transport a sphere from box 1 to box 2), it is found from Eq. (15) that

$$
\mathbf{F}(\mathbf{r}, t)=-\nabla \nu(\mathbf{r}, t)
$$

where the "effective chemical potential" $\nu$ is defined as

$$
\nu \equiv \mu_{c}(\mathbf{r}, t)-\frac{v_{c}^{0}}{v_{s}^{0}} \mu_{s}(\mathbf{r}, t) .
$$

When a colloidal particle is displaced, colloidal material is actually interchanged with an equal volume of solvent [see Fig. 1(b)]. This is the reason why in the effective chemical potential in Eq. (17), the difference between the chemical potential of a colloidal particle and the chemical potential of an equal volume of solvent appears. Consideration of forces acting on a single colloidal particle can also be found in Ref. 24.

From the Gibbs-Duhem relation, it is easily shown that (with $p$ the mechanical pressure)

$$
\delta \nu=\frac{V}{N_{c}} \delta\left\{p-\frac{1}{v_{s}^{0}} \mu_{s}\right\} .
$$

This Gibbs-Duhem relation relates changes of intensive variables, under the condition that the change is established by means of exchanging only reversible work, but no heat, since the temperatures are not changed during these changes. The latter restriction renders the usual contribution $-S \delta T$ on the right-hand side of Eq. (18) absent (with $S$ the entropy). Since $p$ and $\mu_{s}$ are functions of $\left\{N_{c}, N_{s}, V, T\right\}$, Eq. (18) can be written as

$$
\begin{aligned}
\delta \nu= & \frac{V}{N_{c}}\left\{\delta N_{c} \frac{\partial}{\partial N_{c}}+\delta N_{s} \frac{\partial}{\partial N_{s}}+\delta V \frac{\partial}{\partial V}+\delta T \frac{\partial}{\partial T}\right\} \\
& \times\left[p-\frac{1}{v_{s}^{0}} \mu_{s}\right] .
\end{aligned}
$$

In all partial differentiations, $p$ and $\mu_{s}$ are regarded as functions of the independent variables $\left\{N_{c}, N_{s}, V, T\right\}$. Since the addition of $\delta N_{c}$ colloidal particles to a box is accompanied by a decrease of the number of solvent molecules by $\delta N_{s}$ $=-\left(v_{c}^{0} / v_{s}^{0}\right) \delta N_{c}$, it follows immediately that, at constant volume

$$
\delta \nu=\frac{V}{N_{c}}\left\{\delta N_{c}\left[\frac{\partial}{\partial N_{c}}-\frac{v_{c}^{0}}{v_{s}^{0}} \frac{\partial}{\partial N_{s}}\right]+\delta T \frac{\partial}{\partial T}\right\}\left[p-\frac{1}{v_{s}^{0}} \mu_{s}\right] .
$$

Due to the fast adaption of the degrees of freedom of the solvent molecules to the instantaneous distribution of colloidal material and local temperature, one can think of each box as being in osmotic equilibrium with a reservoir of pure solvent of the same temperature and chemical potential as the solvent within the box containing the dispersion. The GibbsDuhem relation for the pure solvent, under the restriction that only reversible work is exchanged, reads (with $p_{s}$ the mechanical pressure within the pure solvent)

$$
\delta p_{s}=\frac{1}{v_{s}^{0}} \delta \mu_{s},
$$

where it is used that for the pure solvent $V_{s}^{\tau} / N_{s}^{\tau}=v_{s}^{0}$, where $V_{s}^{\tau}$ is the volume and $N_{s}^{\tau}$ the number of solvent molecules in the reservoir of pure solvent (the superscript " $r$ " stands for "reservoir"). A change of the osmotic pressure $\Pi \equiv p-p_{s}$ (the difference between the mechanical pressure of the suspension and the pure solvent) is therefore equal to

$$
\delta \Pi=\delta\left[p-\frac{1}{v_{s}^{0}} \mu_{s}\right] .
$$


Note that the change $\delta \mu_{s}$ includes changes due to temperature variations. We shall therefore introduce the variables $s$ on which $\mu_{s}$ depends, besides the temperature

$$
\mu_{s} \equiv \mu_{s}(T, s) .
$$

Furthermore, the operator within the square brackets in Eq. (20) is nothing but $\partial(\cdots) / \partial N_{c}$ for the osmotic system, since one colloidal sphere is always exchanged with $-v_{c}^{0} / v_{s}^{0}$ solvent molecules. Hence, Eq. (20) can be written simply as

$$
\begin{aligned}
\delta \nu & =\frac{V}{N_{c}}\left\{\delta N_{c} \frac{\partial \Pi}{\partial N_{c}}+\delta T \frac{\partial \Pi}{\partial T}\right\} \\
& =\delta \rho \frac{1}{\rho} \frac{\partial \Pi\left(\rho, T, \mu_{s}\right)}{\partial \rho}+\delta T \frac{1}{\rho} \frac{\partial \Pi\left(\rho, T, \mu_{s}(T, s)\right)}{\partial T} .
\end{aligned}
$$

In the last equation it is used that $\Pi$, being an intensive variable, depends on the extensive variables $N_{c}$ and $V$ only through the combination $\rho=N_{c} / V$. Replacing $\delta$ 's by gradients as $\delta(\cdots)=\mathbf{L} \cdot \nabla(\cdots)$, as before, is thus found from Eqs. (16) and (24) that

$$
\begin{aligned}
\mathbf{F}(\mathbf{r}, t)= & -\frac{1}{\rho} \frac{\partial \Pi\left(\rho, T, \mu_{s}\right)}{\partial \rho} \nabla \rho(\mathbf{r}, t) \\
& -\frac{1}{\rho} \frac{\partial \Pi\left(\rho, T, \mu_{s}(T, s)\right)}{\partial T} \nabla T(\mathbf{r}),
\end{aligned}
$$

where the temperature differentiation also acts on $\mu_{s}$. Note that this is equivalent to

$$
\rho \mathbf{F}(\mathbf{r}, t)=-\nabla \Pi(\mathbf{r}, t) .
$$

The body force $\rho \mathbf{F}$ is thus the result of gradients in the osmotic pressure, similar to molecular systems in the absence of flow, where the body force is equal to the gradient of the mechanical pressure. In osmotic systems, work is done against gradients in the osmotic pressure. From Eq. (25) for the force and Eqs. (9)-(11), to leading order in gradients, the equation of motion (3) is recovered with

$$
\begin{aligned}
& D=D_{0} \beta \frac{\partial \Pi\left(\rho, T, \mu_{s}\right)}{\partial \rho}, \text { and } \\
& D_{T}=D_{0} \beta \frac{\partial \Pi\left(\rho, T, \mu_{s}(T, s)\right)}{\partial T},
\end{aligned}
$$

where $D_{0}=k_{B} T / \gamma$ is Einstein's translational diffusion coefficient for a noninteracting sphere (with $k_{B}$ Boltzmann's constant and $T$ the temperature), and $\beta=1 / k_{B} T$. In deriving Eq. (27), terms like $\sim \nabla \rho \cdot \nabla T$ and $\sim \nabla T \cdot \nabla T$ have been neglected. These terms are of second order in deviations of density and temperature from their mean values. Hence, Eq. (3) is valid only to leading order in gradients and small deviations from mean values. The expression (27) for $D$ is a well-known result for the zero wave vector limit of the collective diffusion coefficient, with the neglect of hydrodynamic interactions (see for example, Refs. 24, 28, and 25). Hydrodynamic interactions can be included on the basis of microscopic equations of motion, which will be discussed in the subsequent paper.

Note that in the neighborhood of a gas-liquid critical point, $\beta \partial \Pi / \partial \rho$ is very small. As a result, thermodiffusion becomes particularly pronounced on approach of the critical point, since $\partial \Pi / \partial T$ is well-behaved right up to the critical point. This is also the case on approach of the gas-liquid critical point of molecular mixtures (see Refs. 29, 30, and references therein).

The expressions (27) are valid within a reference frame where there is no volume flux. For incompressible systems this is the usual laboratory frame, where the container does not move with respect to the observer. A factor $1 /(1-\varphi)$ should be added to the right-hand side of Eq. (27) for $D$, when $\Pi$ is expressed as a function of $\rho, T$, and the mechanical pressure $p$, instead of the chemical potential $\mu_{s}$ (where $\varphi$ is the volume fraction of colloidal spheres).

In a one-component fluid, the transport coefficient that describes heat transport due to pressure differences at constant temperature (the so-called mechanocaloric effect) can be related to the thermodiffusion coefficient, using Onsager's symmetry relations. ${ }^{31}$ The mechanocaloric effect for colloidal suspensions, however, is probably primarily determined by the properties of the solvent in which the colloids are embedded. There is therefore no analogous relation between the two transport coefficients for colloids.

\section{THE OSMOTIC PRESSURE AND POTENTIAL OF MEAN FORCE}

An important feature for the thermodiffusive behavior of macromolecular systems is that the interaction potential of an assembly of macromolecules is a potential of mean force, which is implicitly temperature dependent. Although the relation between the osmotic pressure and the potential of mean force is well known, I could not find a comprehensive derivation in literature. Furthermore, the community of researchers dealing with thermodiffusion might not be so familiar with the notion of potential of mean force. I therefore chose to deal with the definition of the potential of mean force and its connection to the osmotic pressure in detail. This connection allows for an explicit evaluation of the transport coefficients $D$ and $D_{T}$ in terms of interaction potentials.

For an osmotic system, where solvent molecules can be exchanged but the number of colloidal spheres is fixed, the appropriate partition function is the semigrand canonical partition function

$$
\begin{aligned}
& \Omega\left(N_{c}, V, T, \mu_{s}\right) \\
& =\frac{\Lambda_{c}^{-3 N_{c}}}{N_{c} !} \sum_{N_{s}=0}^{\infty} \frac{\Lambda_{s}^{-3 N_{s}}}{N_{s} !} \exp \left\{-\beta N_{s} \mu_{s}\right\} \\
& \quad \times \int d \mathcal{R} \int d \omega \exp \left\{-\beta E\left(\mathcal{R}, \omega, N_{s}, N_{c}, V\right)\right\},
\end{aligned}
$$

where $\mathcal{R}$ denotes the set of position coordinates $\left\{\mathbf{r}_{1}, \ldots, \mathbf{r}_{N}\right\}$ of the colloidal spheres, and $\omega$ the spatial and orientational phase space coordinates of the solvent molecules. Furthermore, $E\left(\mathcal{R}, \omega, N_{s}, N_{c}, V\right)$ is the potential energy of the mixture of solvent molecules and colloidal spheres. This "bare potential" is independent of temperature. The de Broglie wavelengths $\Lambda_{s}$ and $\Lambda_{c}$ result from integration of exp $\left\{-\beta E_{\text {kin }}\right\}$ (where $E_{\text {kin }}$ is the total kinetic energy) with respect 
to the momentum coordinates, and from the fact that the smallest possible volume in phase space is equal to $h^{3}$, with $h$ Planck's constant. The solvent- and colloid-de Broglie wavelengths are, respectively, given by

$$
\Lambda_{s}=h / \sqrt{2 \pi m k_{B} T}, \quad \Lambda_{c}=h / \sqrt{2 \pi M k_{B} T},
$$

where $m$ and $M$ are the mass of a solvent molecule and a colloidal sphere, respectively. The grand canonical probability density function $P_{0}$ for $\left\{\omega, N_{s}\right\}$ of pure solvent is equal to

$$
\begin{aligned}
P_{0}\left(\omega, N_{s} \mid \mu_{s}, V, T\right) & \\
= & \frac{\Lambda_{s}^{-3 N_{s}}}{N_{s} !} \frac{\exp \left\{-\beta N_{s} \mu_{s}\right\} \exp \left\{-\beta E_{0}\left(\omega, N_{s}, V\right)\right\}}{\Theta_{0}\left(\mu_{s}, V, T\right)},
\end{aligned}
$$

where the grand canonical partition function $\Theta_{0}$ is given by

$$
\begin{aligned}
\Theta_{0}\left(\mu_{s}, V, T\right)= & \sum_{N_{s}=0}^{\infty} \frac{\Lambda_{s}^{-3 N_{s}}}{N_{s} !} \exp \left\{-\beta N_{s} \mu_{s}\right\} \\
& \times \int d \omega \exp \left\{-\beta E_{0}\left(\omega, N_{s}, V\right)\right\},
\end{aligned}
$$

with $E_{0}$ the potential energy of the pure solvent. The potential of mean force $\Phi$ is now defined as

$$
\begin{aligned}
\exp \{- & \left.\beta \Phi\left(\mathcal{R}, N_{c}, V, T, \mu_{s}\right)\right\} \\
\equiv & \sum_{N_{s}=0}^{\infty} \int d \omega P_{0}\left(\omega, N_{s} \mid \mu_{s}, V, T\right) \\
& \quad \times \exp \left\{-\beta\left[E\left(\mathcal{R}, \omega, N_{c}, N_{s}, V\right)-E_{0}\left(\omega, N_{s}, V\right)\right]\right\} .
\end{aligned}
$$

It is easily seen that $-\nabla_{i} \Phi$ (with $\nabla_{i}$ the gradient operator with respect to the position coordinate of the $i$ th colloidal sphere) is the average of the force $-\nabla_{i} E$ on the sphere over the solvent phase space coordinates with respect to the probability density function

$$
\begin{aligned}
P\left(\omega, N_{s}\right)= & \frac{\Lambda_{s}^{-3 N_{s}}}{N_{s} !} \exp \left\{-\beta N_{s} \mu_{s}\right\} \\
& \times \exp \left\{-\beta E\left(\mathcal{R}, \omega, N_{c}, N_{s}, V\right)\right\} / \mathcal{N},
\end{aligned}
$$

where the normalization constant is equal to

$$
\begin{aligned}
\mathcal{N}= & \sum_{N_{s}=0}^{\infty} \frac{\Lambda_{s}^{-3 N_{s}}}{N_{s} !} \\
& \times \int d \omega \exp \left\{-\beta N_{s} \mu_{s}\right\} \exp \left\{-\beta E\left(\mathcal{R}, \omega, N_{c}, N_{s}, V\right)\right\} .
\end{aligned}
$$

This is the probability density function for the solvent phase space coordinates with a prescribed configuration of colloidal particles. Hence, $-\nabla_{i} \Phi$ is the force on the $i$ th colloidal sphere, averaged with respect to the solvent degrees of freedom for a given configuration of colloidal particles. This is the reason why $\Phi$ is referred to as a "potential of mean force."
It will turn out to be essential for thermodiffusion of a certain class of systems that the potential $\Phi$ is temperature dependent, due to averaging with respect to the solvent degrees of freedom.

Defining the canonical colloid partition function $\Omega_{c}$ that corresponds to the potential of mean force as

$$
\begin{aligned}
\Omega_{c}\left(N_{c}, V, T, \mu_{s}\right) \equiv & \frac{\Lambda_{c}^{-3 N_{c}}}{N_{c} !} \\
& \times \int d \mathcal{R} \exp \left\{-\beta \Phi\left(\mathcal{R}, N_{c}, V, T, \mu_{s}\right)\right\},
\end{aligned}
$$

allows the following, exact factorization of the semigrand canonical partition function (28):

$$
\Omega\left(N_{c}, V, T, \mu_{s}\right)=\Theta_{0}\left(\mu_{s}, V, T\right) \Omega_{c}\left(N_{c}, V, T, \mu_{s}\right) .
$$

The probability density function (pdf) of the position coordinates $\mathcal{R}$ of the colloidal spheres is now equal to

$$
P\left(\mathcal{R} \mid N_{c}, V, T, \mu_{s}\right) \equiv \frac{\Lambda_{c}^{-3 N_{c}}}{N_{c} !} \frac{\exp \left\{-\beta \Phi\left(\mathcal{R}, N_{c}, V, T, \mu_{s}\right\}\right.}{\Omega_{c}\left(N_{c}, V, T, \mu_{s}\right)} .
$$

The expressions (35) and (37) show that the osmotic system can be regarded as an effective canonical system where the potential energy is now equal to the temperature-dependent potential of mean force. In the microscopic approach to thermodiffusion discussed in the subsequent paper, an equation of motion for the corresponding nonequilibrium pdf is analyzed.

From Eq. (36), the pressure is found to be equal to

$$
p=k_{B} T\left[\frac{\partial \ln \{\Omega\}}{\partial V}\right]_{N_{c}, T, \mu_{s}}=p_{s}+\Pi
$$

where the pressure $p_{s}$ of the pure solvent is equal to

$$
p_{s}=k_{B} T\left[\frac{\partial \ln \left\{\Theta_{0}\right\}}{\partial V}\right]_{T, \mu_{s}}
$$

and the osmotic pressure $\Pi=p-p_{s}$ is equal to

$$
\Pi=k_{B} T\left[\frac{\partial \ln \left\{\Omega_{c}\right\}}{\partial V}\right]_{N_{c}, T, \mu_{s}} .
$$

The osmotic pressure is thus related to the potential of mean force in exactly the same way as the mechanical pressure of a canonical molecular system is related to its potential energy.

Assuming a pairwise additive potential of mean force, that is (with $r_{n m}=\left|\mathbf{r}_{n}-\mathbf{r}_{m}\right|$, where $\mathbf{r}_{j}$ is the position coordinate of the $j$ th colloidal sphere)

$$
\Phi\left(\mathbf{r}_{1}, \mathbf{r}_{2}, \ldots, \mathbf{r}_{N} \mid T\right)=\sum_{n<m}^{N_{c}} V\left(r_{n m} \mid T\right),
$$

where $V$ is the pair-interaction potential of mean force, a standard procedure to evaluate Eq. (40) leads to (see, for example Ref. 32) 


$$
\begin{aligned}
\Pi\left(\rho, T, \mu_{s}\right)= & \rho k_{B} T-\frac{2 \pi}{3} \rho^{2} \\
& \times \int_{0}^{\infty} d R R^{3} g^{e q}(R \mid \rho, T) \frac{d V(R \mid T)}{d R},
\end{aligned}
$$

where $R$ is the distance between two colloidal spheres. Here and in the following, we shall only denote the position and temperature dependence of potentials for brevity. Note that the temperature dependence of the pair-potential includes the temperature dependence of the chemical potential of the solvent. The equilibrium pair-correlation function $g^{e q}$ is defined as

$$
g^{e q}\left(R=r_{12} \mid \rho, T\right) \equiv V^{2} \int d \mathbf{r}_{3} \cdots \int d \mathbf{r}_{N} P\left(\mathcal{R} \mid N_{c}, V, T, \mu_{s}\right),
$$

with $P$ the pdf of the position coordinates $\mathcal{R}=\left\{\mathbf{r}_{1}, \ldots, \mathbf{r}_{N}\right\}$ as given in Eq. (37).

Together with Eq. (27), Eqs. (42) and (43) express the transport coefficients $D$ and $D_{T}$ in terms of the potential of mean force.

The pairwise additivity approximation (41) is a very good approximation for potentials, superimposed on a hardcore repulsion, with a range that is small in comparison to the hard-core radius. Even for longer-ranged potentials (like for charged spheres with a large screening length), however, this assumption is quite accurate.

Within the thermodynamic approach used here, it is not possible to include hydrodynamic interactions between the colloidal spheres. The results obtained here are nevertheless semiquantitatively correct when the pair-interaction potential is very long-ranged repulsive as compared to the size of the core, such as charged spheres at low salt concentration. Such systems can be thermodynamically concentrated but hydrodynamically dilute. For such a system one could use, for example, Ornstein-Zernike expressions for the paircorrelation function in Eq. (42), and use the resulting expression to calculate $D$ and $D_{T}$ from Eq. (27). This will not be pursued in the present paper. The inclusion of hydrodynamic interactions requires a microscopic approach, which is developed in the subsequent paper.

In the following sections we shall evaluate $D$ and $D_{T}$ to leading order in concentration for spheres with a very shortranged attraction, superimposed onto a hard-core repulsion. It will turn out that this type of particles can diffuse to hot regions under certain conditions. The numerical values of the virial coefficients are, however, only qualitatively correct. For quantitative values, hydrodynamic interactions must be accounted for.

\section{LEADING ORDER DENSITY EXPANSION OF $D$ AND $D_{T}$}

To leading order in colloid concentration, $D$ and $D_{T}$ can be expressed in terms of the pair-interaction potential of mean force as defined in Eqs. (32) and (41). For such low concentrations, the pair-correlation function, defined in Eq. (43), is equal to

$$
g^{e q}(R \mid \rho, T)=\exp \{-\beta V(R \mid T)\} .
$$

The temperature dependence here is understood to include the temperature dependence of the chemical potential of the solvent. Substitution of this expression into Eq. (42), and separating off the hard-core contribution to the pair-potential, yields $^{33}$

$$
\begin{aligned}
\Pi\left(\rho, T, \mu_{s}\right)= & \rho k_{B} T\left[1+4 \varphi\left\{\exp \left\{-\beta V_{+}(T)\right\}\right.\right. \\
& -\beta \int_{R>2 a} d R\left(\frac{R}{2 a}\right)^{3} \frac{d V(R \mid T)}{d R} \\
& \times \exp \{-\beta V(R \mid T)\}\} .
\end{aligned}
$$

Here, $\varphi=v_{c}^{0} \rho=(4 \pi / 3) a^{3} \rho$ is the volume fraction of colloidal spheres (with $a$ the radius of the core of the colloidal spheres) and $V_{+}$is the contact value of the pair potential, that is

$$
V_{+}(T)=\lim _{\epsilon \downarrow 0} V(R=2 a+\epsilon \mid T) .
$$

For pure hard-core potentials, $V_{+}(T)=0$, and the integral in Eq. (45) vanishes.

From Eqs. (27) and (45), it is found by differentiation with respect to the density, that up to $\mathcal{O}\left(\varphi^{2}\right)$

$$
\begin{aligned}
D= & D_{0}\left[1+8 \varphi\left\{\exp \left\{-\beta V_{+}(T)\right\}\right.\right. \\
& \left.-\beta \int_{R>2 a} d R\left(\frac{R}{2 a}\right)^{3} \frac{d V(R \mid T)}{d R} \exp \{-\beta V(R \mid T)\}\right\} .
\end{aligned}
$$

The thermal diffusion coefficient can be written as a sum of two contributions

$$
D_{T}=D_{T}^{(0)}+D_{T}^{(i)}
$$

where $D_{T}^{(0)}$ is the thermal diffusion coefficient without the implicit temperature dependence of the osmotic pressure through the potential of mean force, and $D_{T}^{(i)}$ the contribution due to this implicit temperature dependence. These two contributions are found by differentiation of the osmotic pressure with respect to its explicit and implicit temperature dependence. From Eqs. (27) and (45), it is found that

$$
\begin{aligned}
D_{T}^{(0)}= & D_{0} \frac{\rho}{T}\left[1+4 \varphi\left\{\exp \left\{-\beta V_{+}(T)\right\}\left[1+\beta V_{+}(T)\right]\right.\right. \\
& -\beta^{2} \int_{R>2 a} d R\left(\frac{R}{2 a}\right)^{3} V(R \mid T) \frac{d V(R \mid T)}{d R} \\
& \times \exp \{-\beta V(R \mid T)\}\},
\end{aligned}
$$




$$
\begin{aligned}
D_{T}^{(i)}= & -D_{0} \frac{\rho}{T} 4 \varphi\left[\exp \left\{-\beta V_{+}(T)\right\} \beta T \frac{\partial V_{+}(T)}{\partial T}\right. \\
& +\beta T \int_{R>2 a} d R\left(\frac{R}{2 a}\right)^{3}\left\{\frac{\partial}{\partial T} \frac{d V(R \mid T)}{d R}\right. \\
& \left.\left.-\beta \frac{\partial V(R \mid T)}{\partial T} \frac{d V(R \mid T)}{d R}\right\} \exp \{-\beta V(R \mid T)\}\right],
\end{aligned}
$$

where $\partial V(R \mid T) / \partial T$ denotes differentiation with respect to the implicit temperature dependence of the pair potential, including its temperature dependence through the chemical potential of the solvent.

The above leading-order virial expansions of $D$ and $D_{T}$ can be tested experimentally once the pair-interaction potential, including its temperature dependence, has been characterized, although for a quantitative test hydrodynamic interactions should be included. Note that $D$ is the zero wave vector limit of the collective diffusion coefficient, which can be measured independently in a dynamic light scattering experiment.

\section{A MODEL CALCULATION OF $D$ AND $D_{T}$}

Especially simple expressions for $D$ and $D_{T}$ are obtained when the potential that is superimposed onto the hard-core repulsive potential is very short ranged in comparison to the size $2 a$ of the core (with $a$ the radius of a colloidal sphere). There are many practical systems for which the potential for $R>2 a$ is indeed short ranged. Examples are cores coated with short polymer chains in bad solvents for the polymers, leading to a short-ranged attraction, cores like silica with a 3-4 nm thick stabilizing hydration layer at high salt concentration, and systems where van der Waals attractions are important, which are relatively short ranged for somewhat larger colloidal particles. For suspensions with particles that interact through such short-ranged potentials, the particular functional form of the potential is probably irrelevant for their macroscopic behavior. Here, we shall consider the "triangular potential",

$$
\begin{aligned}
V(R \mid T) & =\epsilon(T) \frac{R-2 a-\Delta}{\Delta}, \text { for } 2 a \leqslant R \leqslant 2 a+\Delta, \\
& =0, \text { for } 2 a+\Delta<R,
\end{aligned}
$$

where $\epsilon$ is the contact value of the additional potential, with $\epsilon>0$ for attractive potentials, and where $\Delta$ is its range. The depth of the potential is temperature dependent, being a potential of mean force. The width $\Delta$ of the potential is assumed independent of temperature, which is indeed the case, to a good approximation, for the potentials mentioned above. For example, when the core is coated with polymer chains, the width $\Delta$ is of the order of the thickness of the brush, and $\epsilon$ depends on the quality of the solvent for the polymer coating, which is indeed temperature dependent. We shall assume short-ranged potentials, that is

$$
\Delta / 2 a \ll 1 .
$$

The osmotic pressure is easily obtained from Eq. (45) to leading order in $\Delta / 2 a$
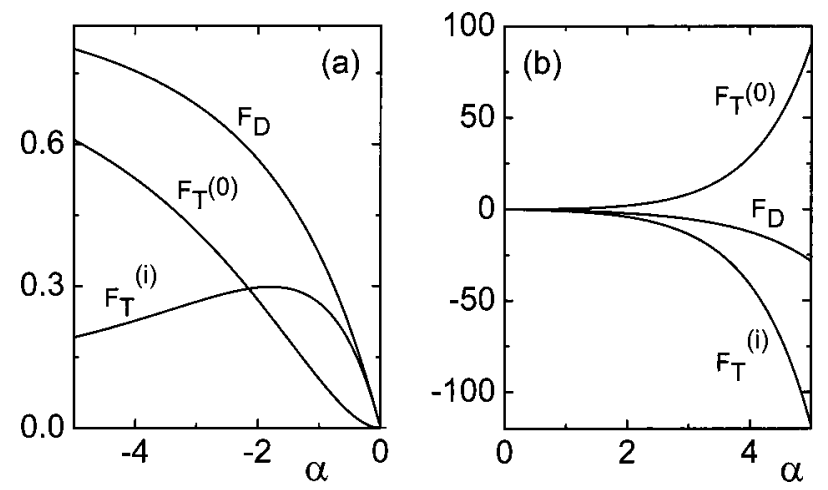

FIG. 2. The functions $F_{D}, F_{T}^{(0)}$, and $F_{T}^{(i)}$, as defined in Eqs. (54), (55), and (56), respectively, for repulsive potentials (a) and attractive potentials (b).

$$
\begin{aligned}
\Pi\left(\rho, T, \mu_{s}\right)= & \rho k_{B} T[1+4 \varphi \\
& \left.\times\left\{1+\frac{3 \Delta}{2 a}\left(\frac{1+\beta \epsilon-\exp \{\beta \epsilon\}}{\beta \epsilon}\right)\right\}\right],
\end{aligned}
$$

where $\varphi$ is the volume fraction of colloidal material. Substitution into Eq. (27) leads to the following expression for the collective diffusion coefficient:

$$
\begin{aligned}
& D=D_{0}\left[1+8 \varphi\left\{1+\frac{3 \Delta}{2 a} F_{D}(\beta \epsilon)\right\}\right], \\
& F_{D}(\alpha)=(1+\alpha-\exp \{\alpha\}) / \alpha .
\end{aligned}
$$

The two explicit and implicit temperature-dependent contributions to the thermal diffusion coefficient in Eq. (48) are equal to

$$
\begin{aligned}
& D_{T}^{(0)}=D_{0} \frac{\rho}{T}\left[1+4 \varphi\left\{1+\frac{3 \Delta}{2 a} F_{T}^{(0)}(\beta \epsilon)\right\}\right], \\
& F_{T}^{(0)}(\alpha)=(2+\alpha+[\alpha-2] \exp \{\alpha\}) / \alpha,
\end{aligned}
$$

and

$$
\begin{aligned}
& D_{T}^{(i)}=D_{0} \frac{\rho}{T} 4 \varphi\left[\frac{T}{\epsilon} \frac{d \epsilon(T)}{d T}\right] \frac{3 \Delta}{2 a} F_{T}^{(i)}(\beta \epsilon), \\
& F_{T}^{(i)}\left(F_{T}^{(i)}(\alpha)=\right. F_{D}(\alpha)-F_{T}^{(0)}(\alpha)=(-1+[1 \\
&-\alpha] \exp \{\alpha\}) / \alpha .
\end{aligned}
$$

The functions $F_{D}, F_{T}^{(0)}$ and $F_{T}^{(i)}$ are plotted for negative values of their argument (repulsive potentials) in Fig. 2(a) and for positive values (attractive potentials) in Fig. 2(b). Note that for deeply attractive potentials, the thermodiffusion functions $F_{T}^{(0)}$ and $F_{T}^{(i)}$ become much larger than the collective diffusion function $F_{D}$. In fact, in the "sticky sphere limit," where $\Delta / 2 a \rightarrow 0$ and $\beta \epsilon \rightarrow \infty$, such that $(\Delta / 2 a) \exp \{\beta \epsilon\} / \beta \epsilon$ remains finite to assure a finite value of $D$, both $D_{T}^{(0)}$ and $D_{T}^{(i)}$ diverge. Thermodiffusion for shortranged potentials therefore cannot be described within the formal sticky sphere limit. For very short-ranged and deeply attractive potentials, there is thus a pronounced Soret effect. For repulsive potentials, the Soret coefficient is positive, as can be seen from Fig. 2(a), while thermodiffusion is not so 

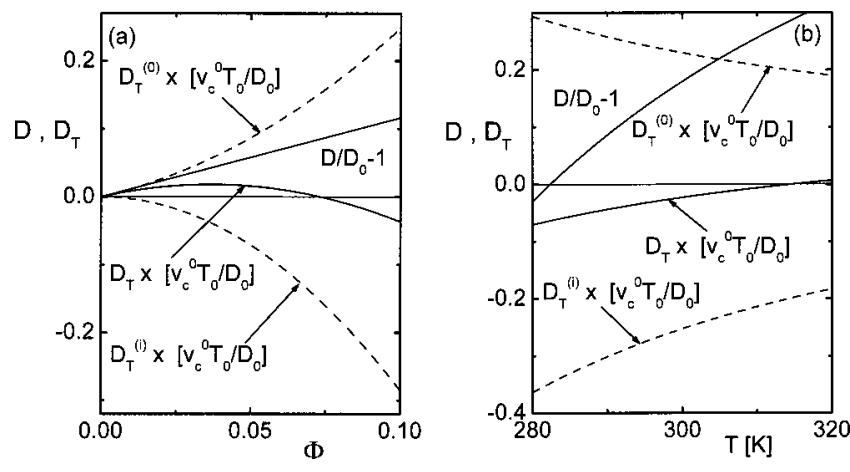

FIG. 3. (a) The various diffusion coefficients in dimensionless form as functions of the volume fraction of colloids, to leading order in concentration. Note that $v_{c}^{0} D_{T} / D_{0}=(\varphi / T)[1+(\cdots) \varphi]$. Here, $\Delta / 2 a=1 / 100$, $d \ln \{\epsilon\} / d \ln \{T\}=10 / \beta \epsilon$ and $\beta \epsilon=5$ at room temperature $T_{0}=293 \mathrm{~K}$. (b) The various diffusion coefficients, again in dimensionless form, as a function of the temperature for $\varphi=0.1$.

pronounced: adding a repulsive potential to the hard-core repulsive potential does not change diffusive properties significantly.

The collective diffusion and thermal diffusion coefficient are plotted in Figs. 3(a) and (b), in dimensionless form, as functions of the volume fraction and temperature, respectively. The parameters are specified in the captions of these figures. For these parameters, $D_{T}$ is negative at large enough concentrations and low enough temperatures. The contribution $D_{T}^{(0)}$ to the thermal diffusion coefficient is always positive. However, when the depth $\epsilon>0$ of an attractive potential increases with temperature (that is, when $d \epsilon / d T>0$ ), the implicit thermal diffusion coefficient $D_{T}^{(i)}$ in Eq. (56) is negative. In that case colloidal particles tend to diffuse to hot regions. The interpretation of this result is, that the potential energy decreases on increasing the temperature, which lowers the free energy. Note that the potential of mean force is not a potential energy in the strict sense: it contains also entropic contributions as a result of averaging with respect to the fast solvent degrees of freedom. The thermal diffusion coefficient $D_{T}=D_{T}^{(0)}+D_{T}^{(i)}$ in Fig. 3 is small in magnitude as compared to both $D_{T}^{(0)}$ and $D_{T}^{(i)}$, which are of opposite sign. There is thus a delicate balance between the explicit and implicit temperature dependence of the osmotic pressure that determines the thermodiffusive behavior of the colloidal spheres.

\section{A COMMENT ON THERMODIFFUSION AT INFINITE DILUTION}

Besides the effects of intercolloidal particle interactions, single-particle properties are important for the overall thermodiffusive behavior of macromolecular systems. Thermodiffusive behavior at infinite dilution can be described on the basis of Eqs. (16) and (17). Changes of chemical potentials are now due to (i) changes of the osmotic pressure for noninteracting particles and (ii) changes due to specific interactions of the surface of the colloidal spheres with solvent molecules. The first contribution follows from Eq. (27), with $\Pi$ equal to the ideal-gas pressure $\rho k_{B} T$. For noninteracting spheres where specific interactions with the solvent are not important, it is thus found that $D_{T}=D_{0} \rho / T$. This "ideal-gas contribution" is already incorporated in the previous analysis. The second contribution, denoted as $\Delta D_{T}$, which has been neglected so far, is related to the temperature dependence of the chemical potential of the "complexed colloidal sphere," that is, the surface groups on the colloidal sphere and its solvation layer together with its ionic cloud (when present). Let $\mu_{\mathrm{cs}}^{*}(T)$ denote the chemical potential of the complexed colloidal sphere (the subscript "cs" stands for "complexed sphere"), where colloid-mixing contributions are not included (as indicated by the "**"). These mixing contributions are already contained in the ideal-gas contribution to $D_{T}$. Following the reasoning that leads to Eqs. (16) and (17) now gives rise to an additional force equal to

$$
\Delta \mathbf{F}(\mathbf{r}, t)=-\nabla \mu_{c s}^{*}(T(\mathbf{r}))=-\frac{\partial \mu_{\mathrm{cs}}^{*}(T)}{\partial T} \nabla T(\mathbf{r}),
$$

and hence, from Eqs. (9)-(11), again to leading order in gradients and deviations of density and temperature from their mean values

$$
\Delta D_{T}=D_{0} \beta \rho \frac{\partial \mu_{c s}^{*}(T)}{\partial T} .
$$

The calculation of $\mu_{c s}^{*}(T)$ requires detailed, molecular knowledge about the interactions between colloidal surface groups and solvent, and possibly its ionic cloud. Alternatively, one might measure $\mu_{\mathrm{cs}}^{*}$ independently, from dispersions so dilute that interactions do not play a role, and subtracting from these data mixing contributions. Assuming that interactions between colloidal particles do not affect $\mu_{c s}^{*}$ too much, the contribution $\Delta D_{T}$ in Eq. (58) should always be added to the ideal gas and interaction contributions to $D_{T}$, the latter to which the present and the subsequent paper are devoted. Hence, in an experimental verification of the effect of interactions on thermodiffusion, experimental values at infinite dilution should be subtracted from those at higher concentrations.

Note that even for an "ideal colloidal gas," inertial forces are not important due to the large friction force with the solvent. This is why the diffusion coefficient $D_{T}$ $=D_{0} \rho / T$ is independent of the mass of the colloidal particles, just as for higher concentrations.

\section{SUMMARY AND CONCLUSIONS}

On the basis of statistical thermodynamics and force balance on the Brownian time scale, expressions for the collective and thermal diffusion coefficient in terms of the osmotic pressure have been derived. There are a number of assumptions made here, which are probably only valid for macromolecular solutions, but not for molecular mixtures. First of all, the force on a macromolecule is obtained by assuming force balance, that is, inertial forces on a colloidal particle are assumed very small. Such a force balance can only be justified when the macromolecule is very much larger than the solvent molecules. ${ }^{24,25}$ Second, molar volumes are taken independent of concentration, temperature, and pressure. This relies on incompressibility and the fact that the molar 
volume of colloids is to a very good approximation equal to the volume of a single colloidal sphere, and that the solvent is predominantly present as bulk material. Third, osmotic equilibrium can be assumed due to the very fast dynamics of the solvent molecules as compared to the colloidal degrees of freedom.

A further special feature of macromolecular systems is that their interaction energy is a temperature-dependent potential of mean force. This implicit temperature dependence can have an important effect on the thermodiffusive behavior of macromolecular systems. For short-ranged attractive potentials for which the depth of attraction increases with increasing temperature, this temperature dependence can lead to "uphill thermodiffusion," that is, diffusion to regions of higher temperature.

The virial expansions of $D$ and $D_{T}$ to leading order in concentration can be tested experimentally. For a quantitative comparison, however, hydrodynamic interactions between the colloidal spheres should be taken into account. For example, for hard spheres (for which $\Delta / 2 a=0$ ), the $8 \varphi$ in Eq. (54) for $D$ is known to change to $1.45 \varphi$ due to hydrodynamic interactions. ${ }^{24,25}$ The leading-order virial coefficient of $D_{T}^{(0)}$ and $D_{T}^{(i)}$ will probably also be affected substantially by hydrodynamic interactions. The present approach does not allow inclusion of hydrodynamic interactions. In order to do this, a microscopic approach to the derivation of the equation of motion (3) for the density must be developed. This is the subject of the subsequent paper.

As discussed briefly in Sec. VI, specific interactions between the surface groups of colloidal spheres and solvent molecules give rise to thermodiffusion due to the temperature dependence of the chemical potential of the complexed colloidal spheres, that is, the colloidal spheres and their solvation layer, and possibly their ionic cloud. This contribution should be subtracted from experimental thermal diffusion coefficients in order to be able to compare the interaction contributions to theoretical predictions.

The interesting colloidal systems of monodisperse, spherical particles are those where the colloidal spheres interact via very short-ranged attractive potentials, where the depth of attraction increases with temperature. For such systems the Soret effect is most pronounced, and sign changes of the Soret coefficient can be expected on variation of concentration, temperature, and solvent properties.

${ }^{1}$ C. Ludwig, Sitzungsber. Kaiser. Akad. Wiss. (Math.-Naturwiss. Kl.), Wien 20, 539 (1856).

${ }^{2}$ Ch. Soret, Arch. Sci. Phys. Nat. 3, 48 (1879), C. R. Hebd. Seances Acad. Sci. 91, 289 (1880), Ann. Chim. Phys. 22, 293 (1881).

${ }^{3}$ L. Onsager, Phys. Rev. 37, 405 (1931).

${ }^{4}$ H. J. V. Tyrrell, Diffusion and Heat Flow in Liquids (Butterworths, London, 1961)
${ }^{5}$ S. R. de Groot and P. Mazur, Non-Equilibrium Thermodynamics (Dover., New York, 1984)

${ }^{6}$ R. J. Bearman, J. G. Kirkwood, and M. Fixman, Adv. Chem. Phys. 1, 1 (1958).

${ }^{7}$ T. N. Khazanovich, J. Polym. Sci., Part C: Polym. Symp. 16, 2463 (1967).

${ }^{8}$ R. F. Streater, Rep. Math. Phys. 40, 557 (1997).

${ }^{9}$ J. Luettmer-Strathmann, J. Chem. Phys. (to be published).

${ }^{10}$ P. F. van Velden, H. G. P. van der Voort, and C. J. Gorter, Physica XII, 151 (1946) (in French).

${ }^{11}$ I. Prigogine, L. de Brouckere, and R. Amand, Physica XVI, 851 (1950) (in French).

${ }^{12}$ C. Debuschewitz and W. Köhler, Phys. Rev. Lett. 87, 055901 (2001).

${ }^{13}$ D. Reith and F. Müller-Plathe, J. Chem. Phys. 112, 2436 (2000).

${ }^{14}$ P. Bordat, D. Reith, and F. Müller-Plathe, J. Chem. Phys. 115, 8978 (2001).

${ }^{15}$ K. I. Morozov, J. Magn. Magn. Mater. 201, 248 (1999).

${ }^{16}$ K. I. Morozov, in Thermal Nonequilibrium Phenomena in Fluid Mixtures, edited by W. Köhler and S. Wiegand (Springer, Berlin, 2002), p. 38.

${ }^{17}$ B.-J. de Gans, R. Kita, B. Müller, and S. Wiegand, J. Chem. Phys. 118, 8073 (2003).

${ }^{18} \mathrm{~S}$. Iacopini and R. Piazza, "Thermophoresis in protein solutions" (unpublished).

${ }^{19}$ E. Blums, S. Odenbach, A. Mezulis, and M. Maiorov, Phys. Fluids 10, 2155 (1998).

${ }^{20}$ R. Piazza and A. Guarino, Phys. Rev. Lett. 88, 208302 (2002).

${ }^{21}$ P. Rossmanith and W. Köehler, Macromolecules 29, 3203 (1996).

${ }^{22}$ J. Rauch and W. Köhler, Phys. Rev. Lett. 88, 185901 (2002).

${ }^{23} \mathrm{~S}$. Wiegand and W. Köhler, in Thermal Nonequilibrium Phenomena in Fluid Mixtures, edited by W. Köhler and S. Wiegand (Springer, Berlin, 2002), p. 189.

${ }^{24}$ G. K. Batchelor, J. Fluid Mech. 74, 1 (1976).

${ }^{25}$ J. K. G. Dhont, An Introduction to Dynamics of Colloids (Elsevier, Amsterdam, 1996).

${ }^{26}$ A change of the internal energy $U$ is equal to, $\delta U=\delta Q^{\text {rev }}+\delta w^{\text {rev }}$, where for a reversible change $\delta Q^{\text {rev }}=T_{1} \delta S_{1}+T_{2} \delta S_{2}$ is the amount of heat added to the two boxes (the index refers to the box). A change in the Helmholtz free energy $F=U-T_{1} S_{1}-T_{2} S_{2}$ is thus equal to $\delta F=-S_{1} \delta T_{1}-S_{2} \delta T_{2}$ $+\delta w^{\text {rev }}$.

${ }^{27}$ There are macromolecules of which the volume can be strongly temperature dependent, such as microgel spheres, colloidal spheres coated with a thick polymer brush of which the thickness varies with temperature and polymers like poly(N-isopropylacrylamide) (PNIPAM). For such systems, the temperature dependence of $v_{c}$ must be included.

${ }^{28}$ C. Van den Broeck and H. N. W. Lekkerkerker, J. Chem. Phys. 74, 2006 (1981).

${ }^{29}$ R. Haase, K.-H. Drücker, H. Buchner, and J. Schwinum, Z. Phys. Chem. (Munich) 186, 113 (1994)

${ }^{30} \mathrm{~J}$. Luettmer-Strathmann, in Thermal Nonequilibrium Phenomena in Fluid Mixtures, edited by W. Köhler and S. Wiegand (Springer, Berlin, 2002), p. 25.

${ }^{31}$ L. Reichl, A Modern Course in Statistical Physics (University of Texas Press, Austin, 1980).

${ }^{32}$ D. A. McQuarrie, Statistical Mechanics (Harper \& Row, New York, 1976), pp. 261-262.

${ }^{33}$ The hard-core part of the pair-potential can be accounted for explicitly as follows. First write $\int_{0}^{\infty} d R(\cdots)=\int_{2 a-\epsilon}^{2 a+\epsilon} d R(\cdots)+\int_{2 a+\epsilon}^{\infty} d R(\cdots)$, with $a$ the core radius and $\epsilon$ an arbitrary small distance. Second, the first integral can be evaluated, noting that $d \exp \{-\beta V\} / d R=\exp \left\{-\beta V_{+}(T)\right\} \delta(R-2 a)$, where $\delta$ is the delta distribution and $V_{+}$the contact value of the pair interaction potential. 